\title{
Subcentros intraurbanos. Teoría y algunas evidencias
}

\section{Intraurban subcenters. Theory and some evidence}

\section{Tito A. Alegría}

Doctor en Planeación y Desarrollo Urbano. Departamento de Estudios Urbanos y del Medio ambiente. El Colegio de la Frontera Norte (El Colef). Carretera escénica Tijuana. Ensenada, Km 18.5, San Antonio del Mar (C.P. 22560) Tijuana, Baja California, México, talegria@colef.mx

Resumen

El objetivo de este artículo es presentar una teoría que explica la localización e intensidad de subcentros (o centros) intraurbanos y una prueba estadística de la misma. A diferencia de estudios anteriores, la teoría aquí presentada propone, primero, definir centro como centro de actividades terciarias $y$, segundo, que la intensidad y localización de los centros depende de factores de oferta y demanda localizada de bienes y servicios, factores que operan de manera conjunta en un medio de mercados imperfectos. Con datos de Tijuana se implementó una comprobación estadística de la teoría, corroborando la mayoría de sus postulados.

Palabras clave: Subcentro; Policentrismo; Teoría del lugar central; Estructura urbana

\section{Abstract}

The aim of this article is to present a theory that explains the location and intensity of the intraurban subcenters (or centers) and a statistical test of it. Unlike previous studies, this article's theory proposes, first, to define a center as a tertiary (trade and service) activities center and, secondly, that the centers' intensity and location depend on factors or characteristics of localized supply and demand for goods and services, factors that operate together in an imperfect markets medium. With data from Tijuana, a statistical test of the theory was implemented, verifying most of its postulates.

Key words: Subcenter; Polycentricity; Central place theory; Urban structure 


\section{INTRODUCCIÓN}

El objetivo de este artículo es presentar una teoría que explica la localización e intensidad de subcentros terciarios intraurbanos, y una prueba estadística de la misma para verificar cuán buena es la teoría respecto de las observaciones (Batty, 2017). Esta investigación se basa en la teoría desarrollada por Alegría (2009), con datos diferentes.

En este artículo se introduce el tema de subcentros revisando selectivamente la literatura, luego se presenta la teoría de esta investigación seguida de un modelo analítico, a continuación van los resultados de la prueba estadística y consecuencias socioespaciales del patrón de centros, y se termina con comentarios que resaltan alcances y limitaciones de este estudio.

\section{ENFOQUES EN EL ESTUDIO DE SUBCENTROS}

El estudio de los subcentros se ha inscrito en dos corrientes intelectuales diferenciadas por su contexto geográfico. La primera desarrollada en América, estudia los subcentros en relación a los usos del suelo intraurbanos y el transporte; la segunda anclada en Europa, los estudia bajo el concepto de policentricidad en tres escalas, intraurbana, interurbana e interregional, predominando la segunda. En ambas corrientes las definiciones de subcentro y policentralidad son diversas, ambiguas o 'estiradas' (van Meeteren, Poorthuis, Derudder y Witlox, 2016; Burger y Evert, 2012).

Dentro de los estudios en América, fue predominante el modelo neoclásico mononcéntrico de usos del suelo por dos décadas (Alonso, 1964; Richardson, 1977). Las críticas al modelo monocéntrico estándar fueron muchas y diversas (Anas, Arnott y Small, 1998; Richardson, 1988) dando cabida a la aparición de modelos teóricos alternativos de usos del suelo para dos o más centros (policentrismo), pero manteniendo el principio de balance entre renta del suelo y costo de transporte en las decisiones de localización. Estos modelos teóricos son de dos tipos (White, 1999). El primero considera a los sub- centros de empleo como dados (determinados exógenamente), es decir, las principales características de la estructura urbana (renta del suelo, densidad, salario) se derivan de centros preexistentes (Sullivan 1986; Wieand 1987; Ross y Yinger, 1995). El segundo determina subcentros endógenamente: los subcentros pueden aparecer en cualquier parte; generalmente consideran la presencia de economías de aglomeración, y algunos introducen a desarrolladores del suelo (Fujita y Ogawa 1982; Henderson y Slade 1993; Anas y Kim, 1996). Ambos tipos de modelos son complejos por lo que generalmente se acude a soluciones numéricas, y la aplicación de este tipo de teorías a datos reales aún no se ha intentado.

En general, estas teorías sugieren que los más importantes determinantes de los subcentros son los costos de transporte al trabajo de las personas, y las ventajas (economías) externas y desventajas (congestión) de la aglomeración, las cuales con el crecimiento de la ciudad tienden a tener un balance negativo en el $\mathrm{CBD}^{1}$ surgiendo balances positivos en otras zonas de la ciudad. Estos modelos policéntricos son teorías que definen subcentro como aglomeración de empleos de todo tipo, no tienen especificaciones operativas de subcentros, y no toman en cuenta la demanda, la cual es uno de los factores principales en la explicación de la localización de las actividades económicas de comercio y servicios en una economía terciarizada.

La dificultad de aplicar los modelos teóricos policéntricos facilitó el surgimiento de definiciones empíricas de subcentros, las cuales consideran al subcentro como centro de empleos, y claman estar auspiciados por teorías policéntricas. La primera definición de subcentro desde esta perspectiva fue la de McDonald (1987). Su procedimiento inicia estimando para cada zona de la ciudad dos indicadores: una densidad bruta de empleo y una razón (tasa) de empleo a población residente, E/R. Esta propuesta ha tenido gran influencia en la definición de subcentros de empleo, tanto en la vertiente del uso de la

1 CBD es el acrónimo en inglés de Central business district (distrito central de negocios de la ciudad). 
densidad de empleos como en la vertiente del uso de la razón $\mathrm{E} / \mathrm{R}$.

Dentro de la primera vertiente, Giuliano y Small (1991) consideran que un centro está conformado por zonas contiguas con densidad mayor a 25 empleos por hectárea. Estos autores influyeron en posteriores intentos similares (McMillen y McDonald, 1998; Cervero y Wu, 1997; Bogart y Ferry, 1999; McMillen, 2001). Las teorías policéntricas, en que se basan estos autores, tienen un esquema segregado de usos del suelo: zonas sin subcentros no tienen empleo. Sin embargo, la mayoría del empleo en cada ciudad está desparramado fuera de los subcentros especificados. Entonces, estos ejercicios empíricos son definiciones sin teoría. Por otro lado, el modo de definir los límites de los subcentros es subjetivo: la definición de subcentro depende del mínimo escogido a priori de densidad o cantidad total de empleos, mínimos que no tienen algún argumento sólido que los respalde.

Bajo esta primera variante, superando la subjetividad al definir límites de los centros, han surgido investigaciones que utilizan análisis exploratorio de datos espaciales y en particular indicadores locales de asociación espacial, respectivamente ESDA y LISA por sus acrónimos en inglés. Centro es definido como el conjunto de zonas contiguas con altos y similares niveles de densidad de empleos (Arribas-Bel y Sanz-García, 2014; Rojas, 2015) o con niveles significativamente mayores que sus vecinos (Redfearn, 2007). Como sus predecesores, estas definiciones de centro son laxas o 'estiradas' por la falta de una teoría.

Dentro de la segunda vertiente que McDonald influenció, Forstall y Greene (1997) definieron como centrales a las zonas que tienen usos del suelo mixto y una razón empleos a residentes (E/R) mayor a 1.25, atrayendo así a personas que vienen desde otras zonas para trabajar. Este procedimiento, aun siendo más realista, tampoco tiene una teoría que lo sustente.

Aunque hubo algunos antecedentes (Alegría, 1997), el estudio de centros en las ciudades de América Latina se ha desarrollado solo en la última década y media (Fernández-Maldona- do, Romein, Verkoren y Parente Paula Pessoa, 2014). De manera similar a los enfoque en Estados Unidos, la mayoría de estos estudios definen centro de manera empírica y como centros de empleo de todo tipo, sin una teoría de localización (Aguilar y Alvarado, 2005; Rodríguez-Gámez y Dallerba, 2012; Gonzales de Olarte y del Pozo Segura, 2012; Galeano, 2013; Usach, Garrido-Yserte y Gallo-Rivera, 2017).

En particular, en el estudio de la zona metropolitana de la ciudad de México se ha derivado una controversia aún no resuelta sobre el patrón espacial de centros también definidos como concentraciones de empleo. Con datos de viajes intraurbanos, se encontró que hay una descentralización generalizada de empleos $y$ viviendas modificando la forma urbana hacia una polinuclear (Graizbord y Acuña, 2005; Graizbord y Santillán, 2005); o ya siendo policéntrica evoluciona a una dispersión tentacular del empleo (Muñiz, Sánchez y García-López, 2015). En contraste, se encontró que el patrón espacial es híbrido aunque predominantemente monocéntrico (Suárez y Delgado, 2009; Suárez, Murata y Delgado, 2016); y con un método que mezcla concentración de actividad económica con diversidad de usos del suelo, se concluyó que hay un débil patrón policéntrico (Montejano, Caudillo y Silván, 2016).

Dado que en las grandes ciudades la mayor parte de los empleos ya no están en su centro tradicional, se propuso que los centros no deben definirse como concentraciones de empleo sino como centros de actividad. Gordon y Richardson (1996) encontraron que en la metrópolis Los Angeles, el $88 \%$ de los empleos estaban fuera de los centros, dispersos, sin revelar una configuración policéntrica, y que la densidad de generación de viajes del comercio era 16 veces mayor que la de manufactura. Esto tiene tres implicaciones. Primero, una zona puede actuar como centro al atraer personas, aún con poca densidad de empleos totales. Segundo, la dispersión del empleo significa que los lugares son centros si son definidos como centros de actividad (incluyendo el consumo final) en vez de centros de empleos. 
Tercero, las áreas urbanas no son caracterizadas por usos del suelo segregados, con actividades económicas en algunos lugares y residencias en otros, sino con usos del suelo mixtos.

Aunque los usos mixtos han sido considerados en estudios de transporte desde hace unas décadas (Manaugh y Kreider, 2013) solo recientemente han sido incluidos en las teorías de usos del suelo. Incorporando la dispersión de empleos y residencias, se han propuesto teorías de estructura urbana que considera usos mixtos a cada distancia del centro principal (Wheaton, 2004), y se ha intentado aplicarla a datos reales solo recientemente (Brinkman, 2016). Este tipo de modelos son un positivo intento de acercarse a la realidad por el lado de la oferta -no consideran el lado de la demanda- a pesar de su complejidad y algunos de sus supuestos no verosímiles.

Algunos estudios indican que los centros intraurbanos presentan una estructura jerárquica (West, Von Hohenbalken y Kroner, 1985; Eaton y Lipsey, 1982; Giuliano y Small, 1991; Bogart y Ferry, 1999; Anderson y Bogart, 2001; Redfearn, 2007), y Richardson (1977) sugirió que esa distribución de empleos pudiera en otra escala seguir a la Teoría del lugar central interurbana de Christaller (publicado originalmente en 1933). En la literatura revisada, el estudio de los centros terciarios de Chicago, Estados Unidos, de Berry (1963) ha sido el único que aplicó de manera directa varios principios de esa teoría al espacio intraurbano, como por ejemplo explicar la jerarquía central solo con el tamaño de la demanda ${ }^{2}$, estimar la demanda a partir de áreas de mercado tangentes delimitadas en el territorio ${ }^{3}$, y asociar el tamaño (población) de esas áreas al número de tipos de actividad (funciones centrales); en contraste, define cen-

2 No tomó en cuenta las variaciones de las características de oferta porque esta teoría considera que todos los negocios del mismo tipo tienen similar tecnología y no aprovechan economías de escala ni las economías externas localizadas, y porque no había datos económicos disponibles de los negocios terciarios observados, utilizando solo salario promedio y empleo total por zona censal.

3 Supone que los mercados no se traslapan en el espacio y que cada actividad en cada centro tiene un área de mercado monopolizada a su alrededor. tros de manera indirecta como los lugares de intersección de calles donde el precio del suelo es alto a lo largo de calles principales. Posteriormente, Berry y Parr (1988) nos advirtieron de los obstáculos y limitaciones de aplicar de manera directa los postulados de esta teoría a la escala intraurbana, principalmente por el traslape de áreas de mercado. Para salvar esta y otras limitaciones al aplicar la Teoría del lugar central al espacio intraurbano se proponen a continuación algunos ajustes correspondientes.

\section{TEORÍA DE TAMAÑO Y DISTRIBUCIÓN DE SUBCENTROS TERCIARIOS}

Considérese como premisa dos hechos. Primero, como la distribución espacial de actividades terciarias es diferente a la de la manufactura, sus factores explicativos debieran ser también diferentes. Segundo, las zonas que contienen actividades terciarias atraen más viajes que el número de personas empleadas y muchos más viajes (por trabajador) que las zonas de manufactura. Considerando estos hechos, esta investigación define centro como un centro terciario ( $\sin$ incluir manufactura), separándose de la práctica de definirlo solo como centro de empleo.

Se propone que en cada gran ciudad las actividades terciarias aparecen formando subcentros organizados jerárquicamente, y que esa organización se puede explicar con el auxilio de la Teoría del lugar central, TLC, en un ambiente de mercados imperfectos (debido a la variación espacial en economías de escala, tecnología, y economías externas). Los agentes económicos tanto de la demanda como de la oferta desarrollan micro comportamientos estratégicos con el fin de reducir costos $y / o$ agrandar las ventas, generando con ello un macro patrón de la estructura de subcentros, un patrón modificado de la TLC. Las restricciones institucionales a la operación de los mercados son mínimas. Como resultado, el patrón de usos del suelo (i.e. el patrón espacial de subcentros) depende principalmente de los mecanismos de mercado. Las políticas de usos del suelo no se oponen a ese patrón o lo siguen. 
Para ajustar la TLC a las particularidades del espacio intraurbano se requieren cinco modificaciones.

a) Tamaño de mercado en vez de área. La TLC propone que el área de mercado de una actividad es igual en cualquier localización y su forma es hexagonal. En las ciudades, sin embargo, esa forma es indefinible porque hay una sobre posición de mercados y los consumidores pueden comprar en zonas diversas.

b) Demanda variable en el espacio. La TLC supone que los consumidores tienen ingresos y densidad iguales, y el tamaño de la demanda depende solo del número de consumidores. En la realidad donde ingreso y densidad son mayores hay mayor demanda, y más actividades se localizan cercanamente.

c) Rendimientos crecientes. La TLC supone competencia perfecta, y que cada actividad tiene similares costos y un solo nivel de ventas igual al mínimo necesario para hacer viable económicamente a la actividad. En realidad, en las ciudades hay rendimientos crecientes (que vienen de economías de escala o mejor tecnología). Como costos y rendimientos varían con la localización, cada actividad presenta diversos niveles de ventas por sobre el mínimo necesario para su viabilidad económica.

d) Ventajas de la aglomeración. La TLC no incluye un modelo explícito del comportamiento de los agentes de la oferta, y la co-localización de actividades diferentes la deriva de razones geométricas más que económicas. En realidad, muchos agentes deciden co-localizarse porque la aglomeración puede reducir costos y aumentar las ventas.

e) Número de actividades en un centro. La TLC supone que la jerarquía central de un centro es equivalente al número de todas las actividades centrales de menor jerarquía más uno. En la ciudad, algunas pocas actividades centrales evitan centros de alta jerarquía.

\section{FACTORES POR EL LADO DE LA DEMANDA}

La localización residencial, el comportamien- to estratégico, y las características sociales y económicas de los consumidores son determinantes de la distribución y tamaño de los subcentros.

El consumidor actúa estratégicamente para reducir costos de transacción. Al elegir dónde comprar, los precios solo son prioritarios como criterio discriminador cuando se compran bienes y servicios de alto precio ( $y$ poca frecuencia de compra) y si la comparación de precios es poco costosa. Los consumidores reducen costos de transacción en cuatro maneras: escogiendo la menor distancia (y tiempo) de viaje de compra, buscando economías de escala al comprar más cantidad de cada producto en cada viaje, buscando economías de alcance al comprar más de un tipo de producto en cada viaje (Stahl, 1987), y comparando precio y calidad de bienes o servicios que son sustitutos cercanos en cada viaje (Schulz y Stahl, 1996; Eaton y Lipsey, 1982). Este comportamiento contribuye a una distribución desigual en el espacio del potencial de compra.

Tamaño y localización de los subcentros están determinados por el potencial de compra de los consumidores, y este depende de tres factores: densidad de población, nivel de ingreso, y preferencias de consumo.

En el caso del ingreso, consumidores con un mayor ingreso pueden comprar más del mismo producto y más tipos de productos, incrementando así tanto sus economías de escala y alcance como la concentración espacial del consumo. En ciudades del Tercer Mundo, como Tijuana, solo las personas de mayores ingresos tienen esa mayor capacidad de concentración. En cualquier ciudad, el nivel de concentración del consumo está limitado por la característica asintótica de la elasticidad ingreso de la demanda: la cantidad consumida de muchos bienes y servicios prácticamente no se incrementa con el ingreso después de cierto nivel de ingreso. Pero este efecto difiere entre ciudades del Primery Tercer Mundo, debido a que las primeras tienen un ingreso promedio mayor y su distribución menos concentrada socialmente (Förster y Pearson, 2002): en el Primer Mundo más grupos sociales tienen una asintótica elasticidad ingreso de la demanda y 
por lo tanto más territorio intraurbano tiene alto potencial de demanda ${ }^{4}$. En una ciudad cuya población mayoritaria es pobre, como Tijuana, el nivel asintótico (casi constante) de la elasticidad ingreso de la demanda corresponde a la minoría de población que tiene el mayor ingreso, los cuales, por residir agrupados, generan un solo gran centro terciario de alta jerarquía en las cercanías. En el resto del territorio, la jerarquía central debiera ser también concomitante con el nivel de ingreso de los residentes.

En el caso de las preferencias de consumo, las personas con ingresos similares probablemente tengan similar habitus (en el sentido de Bourdieu, 1990) y, con ello, similares preferencias de consumo, demandando un conjunto limitado de tipos de bienes y servicios. En zonas habitadas por personas con habitus diversos, es posible la concentración de consumo de diversos tipos de bienes y servicios. Si los centros se definen por su diversidad de actividades -además de su capacidad de atraer personas residiendo en otras zonas-, las zonas con mayor diversidad de residentes tienen un mayor potencial de actuar como centros. La diversidad de una zona se puede representar con un indicador de segregación socio-residencial. Bajos niveles de segregación (alta diversidad de población) generan altos niveles de diversidad de actividades, y con ello su centralidad crece.

La presencia de asentamientos informales en un barrio actúa como variable interviniente para reducir su grado de centralidad de dos maneras. Primero, asentamientos informales recientes concentran habitantes de bajos ingresos. Segundo, generalmente estos asentamientos en una zona limitan la cantidad de suelo para actividades terciarias, así como su accesibilidad por su reducida conectividad. Con el paso del

\footnotetext{
4 Los bienes y servicios vendidos en subcentros tienen una elasticidad de demanda mayor en países pobres que en los ricos (Parkin, Esquivel y Ávalos, 2006). Seale, Regmi y Bernstein (2003) estimaron la elasticidad del ingreso para nueve categorías agregadas de consumo en varios países y encontraron que en todas las categorías las elasticidades mexicanas fueron más altas que en Estados Unidos (seis veces más alta en el caso de comida, bebidas y tabaco).
}

tiempo, sus habitantes eventualmente mejorarán sus ingresos, pero podrían seguir careciendo de suelo para usos terciarios necesario para la formación de subcentros.

\section{FACTORES POR EL LADO DE LA OFERTA}

Los negocios de mayor jerarquía, buscando maximizar ventas ${ }^{5}$, estratégicamente se localizan cerca de las concentraciones de consumo, cerca de los consumidores con mayor ingreso y de zonas con mayor diversidad y densidad de población para que sus ventas sean mayores. Bajando en la jerarquía, los negocios maximizan sus ventas donde el nivel de la demanda cercana sea la correspondiente (los negocios cercanos son atractivos a los consumidores porque les permiten ahorro en dinero y tiempo de transporte). Al mismo tiempo, las actividades prefieren localizarse de manera aglomerada para obtener menores costos y mayores ventas. Estos objetivos de localización se buscan a través de tres estrategias: economías de escala, mejor tecnología, y complementariedad entre actividades diferentes.

Para una tecnología dada, el incremento de la escala de ventas reduce costos unitarios por dos razones. Primero, los costos fijos (infraestructura, equipo, etc.) aumentan menos rápidamente que las ventas. Segundo, hace posible modificar la organización de las ventas y los servicios para incrementar la especialización del personal en tareas específicas, y así acrecentar la productividad. Conseguir economías de escala depende de tener un mayor volumen de ventas. En el espacio urbano, las ventas son mayores en zonas con la mayor concentración del consumo potencial (i.e. población con mayores ingresos, etc.). Al mismo tiempo, economías de escala permiten a un negocio reducir precios de venta atrayendo

5 Desde que Baumol (1959) propuso que algunas firmas tienen como objetivo de producción maximizar ventas en vez de maximizar beneficios se inició al respecto una controversia teórica la cual se opacó con el auge de la corriente neoclásica desde la década de 1980, prevaleciendo el supuesto de maximización del beneficio. Desde hace pocos años, sin embargo, el supuesto de maximización de ventas como objetivo de producción (entre otros objetivos) ha resurgido en la literatura (Mert, 2018). 
a consumidores desde zonas alejadas, impulsando así la centralidad de su zona. Mayores economías de escala en una zona conducen a una mayor concentración de la actividad y un mayor tamaño de mercado, incrementando la jerarquía central de ese subcentro. El nivel de economías de escala varía en el espacio, y es mayor en las zonas más centrales -cerca de los barrios con residentes de alto ingreso-, permitiéndoles tener precios más bajos que las zonas menos centrales. De esta manera, los incentivos de mercado hacen que la redistribución espacial del ingreso personal sea regresiva.

La concentración del consumo potencial también crea incentivos para incrementar ventas adoptando una tecnología diferente y más productiva. Similar a las economías de escala, la introducción de una nueva tecnología incrementa la productividad (ventas mensuales por empleado) y necesita mayor mercado, incrementando la centralidad.

La co-localización (aglomeración) de actividades de diferente tipo también impulsa la jerarquía central de una actividad y de una zona. Los negocios se co-localizan para incrementar ventas incentivados por el comportamiento estratégico de los consumidores (comparación y una parada), y al reducir costos (dinero y tiempo) logísticos cuando compran insumos. Las actividades más sensibles a estos ahorros son las que tienen un menor tamaño mínimo de operación o las más interdependientes. Ambos tipos de incentivos a la co-localización generalmente aparecen interrelacionados en el espacio. Las ventajas que una firma consigue por estar localizada junto a actividades de otro tipo, incrementa la densidad de establecimientos del conjunto de las actividades terciarias en la zona.

\section{DEFINICIONES Y MODELO ANALÍTICO}

Para diseñar el modelo de análisis de la teoría expuesta primero se define centro (o subcentro); para ello derivamos la especificación de actividad central (sectorial) y luego de centro (lugar). Para especificar la jerarquía de una actividad, esta investigación usa el tamaño de mercado en lugar del área de mercado, y al sector terciario lo divide en i actividades económicas a escala de rama; además, propone usar el indicador de empleo o cantidad de personal $(E)$, considerando la posibilidad de que, entre todos los indicadores censales, empleo sea el más preciso.

\section{Centro y GRADO dE CENTRALIDAD (GC)}

El tamaño de mercado de una actividad i localizada en la zona j no se puede calcular con datos censales, pero puede ser estimado en términos de la proporción de la población de la ciudad que va a j como consumidores. Con este objetivo, se divide el número de empleados de la actividad $i$ en zona $j\left(E_{i j}\right)$ en dos partes: una cantidad $E_{L i j}$, la cual es el empleo en $\mathrm{j}$ que serviría a la población que reside en j; y una cantidad $E_{x i j}$, la cual es el empleo en $\mathrm{j}$ que serviría a residentes de zonas diferentes a $\mathrm{j}$ que vienen a consumir en j. Aquí suponemos que la balanza comercial interurbana por actividad es cero; más precisamente, se supone que los empleos que cualquier zona j pierde por el consumo de residentes locales en otra ciudad (importación) son compensados por el consumo de foráneos que vienen de otra ciudad (exportación). Este supuesto es verosímil si consideramos que los foráneos tienden a consumir en las zonas más centrales y los locales que salen a consumir (por ejemplo, a San Diego) podrían consumir también en esas mismas zonas más centrales, como veremos más adelante. Entones, el empleo de la actividad i en zona j sería:

$$
E_{i j}=E_{L i j}+E_{x i j}
$$

Si $\mathrm{E}_{x \mathrm{ij}}>0$, la actividad $\mathrm{i}$ en $\mathrm{j}$ atiende también a personas que vienen desde otras zonas. $\mathrm{Si}_{\mathrm{xij}}=0$, $E_{L i j}$ sería suficiente empleo para servir a la población en $j\left(P_{j}\right)$ como $E_{i}$ es suficiente para servir a la población de toda la ciudad $\left[\left(E_{L i j} / P_{j}\right)=\left(E_{i} / P\right)\right]$, bajo el supuesto de que la capacidad de consumo promedio en j es similar a esa capacidad de toda la ciudad. Pero este supuesto no es realista porque el consumo promedio varía entre zonas. 
Para levantar este supuesto, la capacidad de consumo promedio en j debiera estar explícita, y ello se puede expresar de la siguiente manera:

$$
\frac{E_{L i j}}{P_{j}\left(Y_{L i j} \Phi_{L i j}\right)}=\frac{E_{i}}{P(Y \Phi)}
$$

donde $Y_{L i j}$ con $\Phi_{L i j}$ son respectivamente el ingreso promedio (pesos) y la propensión al consumo promedio de $P_{j}$ (sin unidades), además $Y$ con $\Phi$ son respectivamente el ingreso promedio y la propensión al consumo promedio de toda la ciudad. El lado derecho de (2) es una constante.

Trasponiendo términos:

$$
\frac{E_{L i j}}{P_{j}}=\frac{Y_{L i j} \Phi_{L i j}}{Y \Phi} \frac{E_{i}}{P}
$$

el incremento del ingreso del consumidor, es decir, hay satisfacción decreciente con el incremento del consumo, y entonces la propensión al consumo cae con el incremento del ingreso; se supone que esa elasticidad tiende a ser asintótica con los valores del ingreso, ello significa que cuando el ingreso crece la propensión al consumo tiende generalmente a decrecer a proporciones cada vez más pequeñas hasta que la propensión en los rangos medios y altos de ingreso prácticamente se hace constante cuando el valor del ingreso crece.

Trasponiendo términos en la ecuación (1) y reemplazándolos en la ecuación (4) tenemos:

$$
\frac{E_{i j}-E_{x i j}}{P_{j}}=\lambda_{L i j} \frac{E_{i}}{P}
$$

la cual puede ser escrita como:

Si hacemos que:

$$
\lambda_{L i j}=\frac{Y_{L i j}}{Y} \frac{\Phi_{L i j}}{\Phi}
$$

$$
E_{x i j}=E_{i j}-E_{i}\left(\frac{P_{j}}{P}\right) \lambda_{L i j}
$$

podemos reescribir la ecuación (3) como sigue:

$$
\frac{E_{L i j}}{P_{j}}=\lambda_{L i j} \frac{E_{i}}{P}
$$

nótese que $\lambda_{\mathrm{Lij}}$ varía con el ingreso y propensión a consumir promedios en $\mathrm{j}$ expresados como el número de veces sobre (o debajo) del ingreso y propensión promedios (constantes) de toda la ciudad $\left(y_{\mathrm{Lij}}, \varphi_{\mathrm{Lij}}\right)$.

La propensión al consumo es definida como la proporción del ingreso dedicada al consumo de bienes y servicios ofrecidos por la actividad i. El valor de la propensión al consumo es mucho más pequeño que la unidad y varía con la elasticidad-demanda del ingreso, la cual a su vez varía de manera inversa con el ingreso. Aquí se considera que la mayoría de los bienes y servicios tienen una elasticidad decreciente con
La derivación hecha entre las ecuaciones (2) y (6), válida para el empleo que sirve a los consumidores residiendo en la zona analizada, se puede hacer también para el empleo en esa misma zona generado por los consumidores que vienen de otras zonas. Los consumidores que vienen desde fuera de la zona j, que aquí llamamos $\mathrm{P}_{x i \mathrm{j}}$, generan $\mathrm{E}_{x i \mathrm{j}}$ empleos en $\mathrm{j}$. Si suponemos que el consumo efectivo promedio de los $P_{x i j}$ consumidores es el mismo que el consumo efectivo promedio de $\mathrm{P}$ en toda la ciudad, la siguiente razón es cierta:

$$
\frac{E_{x i j}}{P_{x i j}}=\frac{E_{i}}{P}
$$

Sin embargo, la similitud en el consumo promedio de $P_{x i j}$ y $P$ no es realista. Para levantar el supuesto de similitud ponderamos cada población con su consumo efectivo en la ecuación (7): 


$$
\frac{E_{x i j}}{P_{x i j}\left(Y_{X i j} \Phi_{X i j}\right)}=\frac{E_{i}}{P(Y \Phi)}
$$

donde $Y_{x i j}$ es el ingreso promedio de las personas que vienen de fuera a consumir a j, y $\Phi_{\text {xi }}$ es la propensión a consumir promedio de $\mathrm{P}_{x i j}$. $\mathrm{Si}$ hacemos que:

$$
\lambda_{x i j}=\frac{Y_{x i j}}{Y} \frac{\Phi_{x i j}}{\Phi}
$$

entones podemos reescribir la ecuación (8) como sigue:

$$
\frac{E_{x i j}}{P_{x i j}}=\left(\lambda_{x i j}\right) \frac{E_{i}}{P}
$$

Reemplazando $\mathrm{E}_{\mathrm{xij}}$ en la ecuación (8a) con la (6) se obtiene la proporción de población urbana que no vive en $\mathrm{j}$ pero que consume en $\mathrm{j}$ :

$$
\frac{P_{x i j}}{P}=\left(\frac{E_{i j}}{E_{i}}-\frac{P_{j}\left(\lambda_{L i j}\right)}{P}\right) /\left(\lambda_{x i j}\right)
$$

Con la ecuación (9) es posible obtener la proporción total de la población urbana que consume en j ponderada por su consumo efectivo:

$$
\frac{P_{x i j}\left(\lambda_{x i j}\right)+P_{j}\left(\lambda_{L i j}\right)}{P}=\frac{E_{i j}}{E_{i}}
$$

El lado izquierdo de la ecuación (10) es el tamaño de mercado (expresado en términos de proporción de población ponderada por su consumo efectivo) de la actividad i cuando está localizada en j. Con el lado derecho de la ecuación se podría estimar el tamaño del mercado de la actividad i en la zona j de una manera directa con datos del censo económico.
Desarrollando la ecuación (10):

$$
\frac{P_{j}\left[\left(P_{x i j} / P_{j}\right)\left(\lambda_{x i j}\right)+\left(\lambda_{L i j}\right)\right]}{P}=\frac{E_{i j}}{E_{i}}
$$

$$
\left(P_{x i j} / P_{j}\right)\left(\lambda_{x i j}\right)+\left(\lambda_{L i j}\right)=\frac{E_{i j}}{E_{i}} \frac{P}{P_{j}}
$$

$$
\left(\frac{P_{x i j}}{P_{j}}\right) \lambda_{x i j}+\lambda_{L i j}=\frac{\frac{E_{i j}}{P_{j}}}{\frac{E_{i}}{P}}
$$

El lado izquierdo representa al tamaño del consumo de bienes o servicios $i$ en $j$ hecho por su población j y la externa ponderadas por sus respectivas capacidades de consumo relativas (por sobre o debajo) a la capacidad de consumo promedio en la ciudad. Su estimación directa es imposible porque no sabemos la localización y el tamaño de la población $P_{x i j}$ ni los valores de $\lambda_{\text {xij }}$ y $\lambda_{\text {Lij }}$ para cada actividad. El lado derecho de la ecuación (11) expresa el resultado del consumo (lado izquierdo) sobre el empleo de i dentro de j, relativo a la población: el número de veces más en que una actividad $i$ asiste a gente (a consumidores propios y externos) en la zona j en comparación con la asistencia promedio de esa actividad en toda la ciudad. Considerando balanza comercial cero entre ciudades (en cada actividad), podemos de manera equivalente asumir que en cada ciudad todos los empleados de una actividad asisten a todos los habitantes de esa ciudad: un empleado en una actividad de la ciudad es suficiente para asistir a un número específico de habitantes en promedio.

De esta manera, el lado derecho de la ecuación (11) expresa la capacidad de i en j de atraer (o no) consumidores externos; esta capacidad es el nivel de centralidad de la actividad $i$ en la zona j, capacidad que podemos denominar índice de centralidad $C_{i j}$ : 


$$
C_{i j}=\frac{\left(E_{i j} / P_{j}\right)}{\left(E_{i} / P\right)}
$$

donde:

$\mathrm{C}_{\mathrm{ij}}=$ índice de centralidad de la actividad (rama) i en zona $\mathrm{j}$

$E_{i j}=$ empleados de la actividad $\mathrm{i}$ en zona $\mathrm{j}$

$\mathrm{E}_{\mathrm{i}}=$ empleados de la actividad $\mathrm{i}$ en toda la ciudad

$P_{j}=$ población en zona $j$

$\mathrm{P}=$ población en toda la ciudad

Una actividad es definida como central en una zona cuando su índice de centralidad es mayor que la unidad $\left(C_{i j}>1\right)$. Una actividad es no central si le vende su producto solo a una parte de los habitantes de su propia zona, teniendo los restantes habitantes que hacer sus compras en otras zonas donde esa actividad sería central. Este índice de centralidad nos informa sobre la distribución espacial de la centralidad de una actividad a través de j, y sobre con que intensidad a través de $\mathrm{i}$.

Siguiendo a la TLC intraurbana, una zona es definida como central cuando tienen una o más actividades centrales, y su jerarquía central o grado de centralidad, GC $_{j}$, es definida por la cantidad de actividades centrales que la zona contiene:

$$
G C_{j}=\#\left(C_{i j}>1\right)
$$

Con esta definición de centralidad, cualquier zona (o AGEB) puede ser central si su $G C_{j} \geq 1$. Se espera que el grado de centralidad sea mayor en las zonas donde haya mayor actividad del terciario.

\section{Modelo estadístico}

Para la comprobación de la teoría de centros arriba expuesta, se propone un modelo de regresión que explique el grado de centralidad

$G_{\mathrm{j}}$. En el modelo, la jerarquía de centros está determinada de manera conjunta por los indicadores de economías internas y de ventajas externas, y por características de la demanda.

En el modelo hay tres características del consumidor que influencian la jerarquía central por el lado de la demanda: ingresos, densidad, y diversidad de preferencias de la población.

La influencia del ingreso en la generación de centros se prueba con dos variables: i) proporción de personas que reciben de 2 a 5 salarios mínimos, SM; y ii) proporción de personas que reciben más de $5 \mathrm{SM}$. En la exploración del modelo se encontró que el rango bajo de ingreso (proporción de personas que reciben menos de 2 SM) explicaban poco estadísticamente a GC. La densidad de población se especifica como el número de habitantes por hectárea.

El efecto de la diversidad de consumidores en GC se mide con un indicador de segregación socio-espacial. Segregación se estima con Sj que muestra cuán intensa es la mezcla social de un barrio, con cuatro grupos de ingreso agrupados según el número de salarios mínimos (Alegría, 1994):

$$
S_{j}=\sum_{i=1}^{n}\left(\frac{n}{n-1}\right)\left(p_{i j}-\frac{1}{n}\right)^{2}
$$

donde:

$\mathrm{S}_{\mathrm{j}}=$ índice de segregación por ingreso en zona j $(0 \leq S \leq 1)$

$\mathrm{P}_{\mathrm{ij}}=$ proporción de población del grupo de ingreso $\mathrm{i}$ en zona $\mathrm{j}$

$\mathrm{n}$ = número de grupos de ingreso (4 grupos:

0-1 SM, 1-2 SM, 2-5 SM, más que 5 SM)

El rango de este índice va de cero a uno lo que permite una fácil interpretación:

$\mathrm{S}_{\mathrm{j}}=0$, hay nula segregación entre grupos, gran heterogeneidad social en zona j; todos los grupos de ingreso tienen la misma proporción de población en zona j.

$\mathrm{S}_{\mathrm{j}}=1$, hay absoluta segregación entre grupos, total homogeneidad social en zona j; solo un 
grupo de ingreso existe en esta zona.

En la exploración del modelo de regresión, cada variable independiente de demanda tiene 4 variantes, una con datos directos y 3 con su potencial, estimados con el inverso de la distancia afectado por las potencias 1, 1.5 y 2 respectivamente, pero solo una de ellas se incluye en el modelo final: la variable con mayor significancia estadística. Las variables finales a probar en el modelo son los logaritmos naturales de esas 4 variables. A continuación se muestra el ejemplo de la estimación del potencial de Proporción de residentes en rango de ingreso ganando más de 5 salarios mínimos en zona j, $\mathrm{Pl}_{5 \mathrm{j}}$ :

$$
p I_{5 j}=\frac{\sum_{k} \frac{I_{5 k}}{d_{j k}^{\varphi}}}{\sum_{j} \sum_{k} \frac{I_{5 k}}{d_{j k}^{\varphi}}}
$$

$j$ al igual que $k$ son zonas $[j=1,2, . ., n]$ $[k=1,2, . ., n], d$ es distancia en kilómetros desde $j$ hacia cada $k, \phi$ es exponente de distancia $[\phi=1$, $1.5,2]$. A mayor distancia hacia j se reduce la influencia de la característica en zona $k$ sobre una actividad en j. Por ejemplo, cuando $\phi$ es 1 , las personas vienen a consumir en $\mathrm{j}$ de zonas localizadas lejos; cuando $\phi$ es 2 , las personas vienen a consumir en j prácticamente solo de las zonas adyacentes.

Por el lado de la oferta, es imposible medir directamente el efecto de la escala y las externalidades. En consecuencia, se usan características agregadas que son consecuencia de la búsqueda de economías internas y de ventajas externas. Una de ellas es productividad del trabajo (ventas por trabajador, V/E). Como productividad refleja tanto las ventajas internas (economías de escala, tecnología) como externas, se añade la densidad de establecimientos terciarios para que represente sin ambigüedad a las ventajas externas (i.e. cercanía de las firmas a consumidores y proveedores), dejando a la productividad la representación de las economías internas. Como las actividades de comercio aparecen en más zonas que los servicios, es probable que las economías internas y externas operen de manera diferente en estos sectores. Por esta razón, el modelo de regresión incluye dos variables de productividad, una para comercio y otra para servicios.

Con los componentes aquí expuestos, el modelo estadístico de explicación de GC es:

$G C_{j}=\alpha\left(\frac{V_{s j}}{E_{s j}}\right)^{\beta_{1}}\left(\frac{V_{c j}}{E_{c j}}\right)^{\beta_{2}}\left(D e_{j}\right)^{\beta_{s}}\left(p I_{2 j}\right)^{\beta_{2}}\left(p I_{5 j}\right)^{\beta_{s}}\left(p D_{j}\right)^{\beta_{s}}(p S)^{\beta_{1}}(F)^{\beta^{\alpha}}(p C)^{\beta_{3}}$

donde:

$\mathrm{GC}_{\mathrm{j}}=$ grado de centralidad en zona $\mathrm{j}$

Oferta:

$\mathrm{V}_{\mathrm{sj}} / \mathrm{E}_{\mathrm{sj}}=$ productividad (ventas por empleado) de sector servicios en zona $j$

$\mathrm{V}_{\mathrm{cj}} / \mathrm{E}_{\mathrm{cj}}=$ productividad (ventas por empleado) de sector comercio en zona $j$

$D e_{j}=$ densidad de establecimientos terciarios (firmas) en zona $\mathrm{j}$

\section{Demanda:}

$\mathrm{pl}_{2 \mathrm{j}}=$ potencial en zona $\mathrm{j}$ de proporción de residentes que ganan de 2 a 5 salarios mínimos $\mathrm{pl}_{5 \mathrm{j}}=$ potencial en zona $\mathrm{j}$ de proporción de residentes que ganan más de 5 salarios mínimos

$\mathrm{PD}_{\mathrm{j}}=$ potencial en zona $\mathrm{j}$ de la población

$\mathrm{pS}_{\mathrm{j}}=$ potencial en zona $\mathrm{j}$ de la segregación por ingreso

$F_{j}=$ porcentaje de área ocupada por asentamientos originalmente informales

$\mathrm{pC}=$ potencial de longitud $(\mathrm{km})$ de vías en zona j menos la evaluada

$\alpha, \beta 1 \ldots \beta 9=$ constantes de regresión

\section{DATOS}

El estudio se hizo para el área urbana de Ti- 
juana, Baja California. Los datos de las personas provienen del Censo de población y vivienda 2000 y los datos de las actividades económicas provienen de los Censos económicos 2004 (con datos de 2003); datos y mapas provienen de INEGI. Los datos económicos y de población corresponden a años diferentes, por lo que se tomó como base el mapa de datos más reciente donde se descargó los datos menos recientes; esos censos fueron los últimos en que a escala de AGEB (Area geoestadística básica censal) INEGI, respectivamente, preguntó por ingreso de la PEA y del cual provee datos económicos por rama, entonces esos censos son los más recientes con los cuales se puede hacer el análisis que aquí se presenta.

Los datos están a escala de AGEB (polígonos con un área promedio equivalente a un círculo de medio kilómetro de radio aproximadamente); los económicos están a cuatro dígitos de desagregación sectorial (rama). Algunos datos de actividades terciarias de Tijuana no fueron publicados por INEGI debido a que las firmas tienen su sede en otra ciudad, entre ellas están banca, finanzas y comercio de inmuebles (de banca y finanzas no aparece ni un establecimiento). Afortunadamente, esas actividades tienen poca porción del empleo local.

En síntesis, fueron analizadas 128 ramas, 40 de comercio y 88 de servicios. El tamaño del empleo por rama varía fuertemente, y como era de esperarse, el mayor (más de 12 mil empleos) lo tiene la rama 4611 "Comercio al por menor de alimentos". El número de AGEBs en el censo del 2000 fueron 388, y en el censo del 2003 fueron
445, por lo que en la base de datos no hay información de PEA en 57 casos, pero se estimó la población para 2003 interpolando los datos del censo del 2000 y el conteo 2005.

\section{RESULTADOS}

El primer paso fue estimar el Grado de centralidad por barrio, GC, el cual es definido como el número de ramas centrales en cada lugar. La ventaja analítica de este indicador es que se puede estimar con datos censales a escala de AGEB.

En el Mapa 1 y Cuadro 1 se presenta el GC por barrio agrupados en tres rangos de valores ${ }^{6}$. Ningún barrio tuvo más de 75 ramas centrales, y los 17 de mayor rango aparecen formando un solo gran centro lineal de $8 \mathrm{~km}$ de largo. Los 98 barrios en el segundo rango de centralidad (11 a 44 ramas centrales) aparecen en el espacio como centros de jerarquía intermedia en varias partes de la ciudad aunque muy pocos en la periferia. Los 234 barrios de menor jerarquía (1 a 10 ramas centrales) en su mayoría ocupan los espacios entre los de segunda jerarquía. Los 96 barrios sin centro se localizan mayoritariamente en la periferia; entre ellos 49 no tuvieron actividades terciarias.

6 Se puede esperar que haya autocorrelación espacial de GC en varias zonas de la ciudad, pero ello no implica la existencia de dependencia espacial. De acuerdo con la TLC intraurbana, el nivel de GC en un barrio no depende de dicho nivel en el barrio vecino; el valor de GC en cada lugar está determinado por factores estructurales: de oferta a escala de la firma dentro del propio barrio y de demanda (propia y la proveniente de barrios cercanos). GC no se propaga (o contagia por sí misma) en el espacio como lo hace por ejemplo la contaminación del aire desde una fábrica en un barrio sobre barrios vecinos, donde sí habría dependencia espacial de la contaminación. 


\section{Mapa 1. Tijuana. Grado de Centralidad GC}

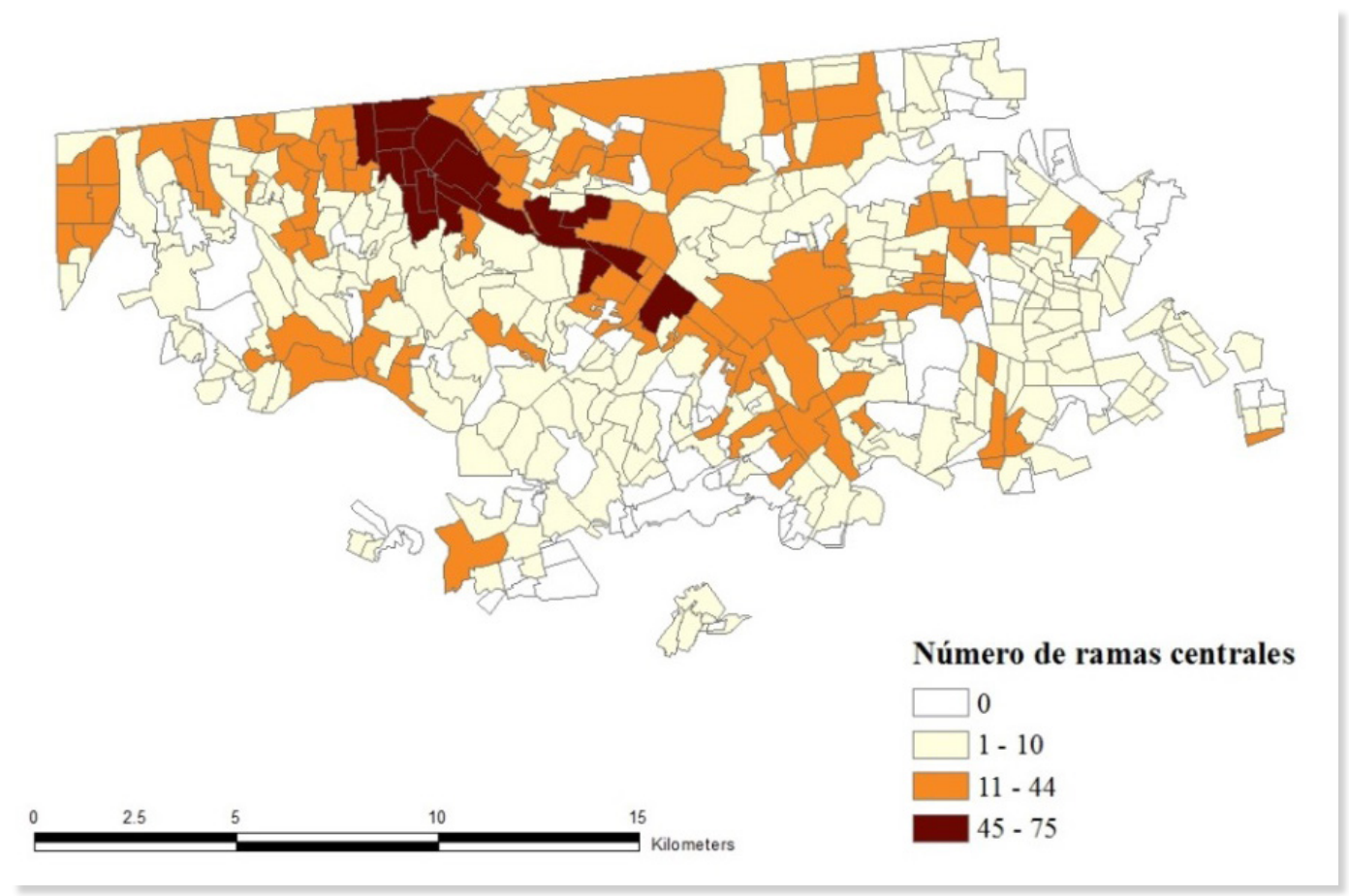

Fuente: elaboración personal con base en datos del Censo de población 2000 y Censos económicos 2004

Cuadro 1. AGEBs y distribución de empleo terciario en tres rangos de GC

\begin{tabular}{lrrrrr}
\multirow{2}{*}{ Rango } & Valor de GC & AGEBs & \% AGEBs & \multicolumn{2}{c}{ \% de PEA (vertical) } \\
\cline { 6 - 7 } & $45-75$ & 17 & 4 & 31 & 44 \\
\hline 2 & $11-44$ & 98 & 22 & 36 & 30 \\
\hline 3 & $1-10$ & 234 & 53 & 29 & 25 \\
\hline Sin centro & 0 & 47 & 11 & 4 & 1 \\
\hline Sin actividad terciaria & & 49 & 11 & 0 & 0 \\
\hline
\end{tabular}

Fuente: elaboración personal con base en datos del Censo de población 2000 y Censos económicos 2004

La distribución de AGEBs por rangos de GC del Cuadro 1 confirma tres proposiciones teóricas de la TLC intraurbana para ciudades latinoamericanas: primero, la distribución es jerárquica a la Christaller con un centro principal y subcentros dispersos de menor jerarquía; segundo, hay pocos lugares de alta jerarquía (4\% de 445 barrios) mientras la mayoría son de menor jerarquía (53\%); y tercero, hay actividades terciarias en $89 \%$ de los barrios y viviendas en el $99 \%$, mostrando que usos mixtos del suelo es el patrón espacial generalizado.
La composición de actividades también varía por rango de centralidad. La actividad comercio se distribuye aproximadamente de manera similar en los tres rangos de GC, con una pequeña sobre representación en el segundo rango. En contraste, los servicios siguen estrictamente la jerarquía central, pues tienen en el más alto rango el $44 \%$ de su empleo en solo $4 \%$ de los barrios, y en el rango más bajo aparece $25 \%$ de su actividad en 53\% de los barrios. Entonces, servicios no solo es la actividad más central sino también la más concentrada espacialmente 
(empleos totales en servicios son solo $10 \%$ más que en comercio). Además, los barrios sin centro contienen $4 \%$ del empleo de comercio pero solo el $1 \%$ de los servicios.

La explicación estadística de la distribución espacial de las intensidades de centros, GC, es el procedimiento de comprobación de la TLC intraurbana arriba expuesta. En la estimación se probaron varios modelos de regresión debido a la presencia de colinealidad entre varias variables y al poco poder explicativo de algunas de ellas (Cuadro 2).

Por el lado de la demanda, la teoría propone que GC crece si en las cercanías hay consumidores con mayor ingreso, diversidad de gustos y con más densidad. Los resultados muestran que los dos primeros factores son relevantes en el modelo pero no así la densidad de población (Modelo 3). Hay más GC: si hay mayor proporción de personas que gana más de 5 salarios mínimos (SM) en las cercanías medidas con el potencial de la variable con exponente $\Phi$ igual a 1 sobre el inverso de la distancia (P1), y si hay menor proporción de personas que gana entre 2 y $5 \mathrm{SM}$ en las cercanías medida con el potencial de la variable con exponente igual a 2 sobre el inverso de la distancia (P2). La diversidad de preferencias de consumo (medido con la segregación) también es determinante en el modelo: a menor segregación en el barrio habrá mayor GC; entonces, la centralidad crece si en el barrio residen personas diversas por sus ingresos y gustos. La irrelevancia en el modelo de la densidad de población, por su parte, se puede deber a que la densidad no tiene un patrón espacial definido, teniendo niveles altos en barrios cuya localización depende de factores institucionales como es el caso de los densos conjuntos edificados por el INFONAVIT hace unas décadas.

Al implementar la regresión con oferta y demanda de manera conjunta, no entran al modelo las variables Productividad Servicios, Segregación y Densidad de población (Modelo 4). Como ya se expuso, solo la densidad no tiene relación con el grado de centralidad; las dos primeras variables si tienen esa relación, pero no entran debido a que su poder explicativo no es muy alto y presentan colinealidad con las otras variables. Este modelo conjunto explica a GC en $57 \%$ (R2), mostrando que la estructuración de centros es más complejo que el modelo: la teoría, siendo aceptable, necesita agregar otros determinantes de la distribución espacial de las intensidades de la centralidad.

Por el lado de la oferta, la teoría propone que GC depende internamente de economías de escala y variación tecnológica, y externamente de las ventajas de la co-localización. Mayor escala y mejor tecnología incrementan la productividad de las actividades de un lugar a condición de que expandan su mercado, es decir su GC; las estimaciones muestran que la productividad de comercio y servicios tienen gran significancia estadísticas (Modelo 1). Las ventajas externas, medidas con la densidad de establecimientos, constituyen el factor más importante en la determinación de GC, de manera que debilitan en el modelo al factor productividad de servicios que tiene menor poder explicativo y algo de colinealidad con las otras dos variables (Modelo 2).

Desde el modelo monocéntrico, a la accesibilidad -a través del costo de transporte-, se le ha considerado determinante de la concentración de actividades económicas en el espacio urbano. Medida con la longitud de vías principales que atraviesan cada barrio, la accesibilidad tiene una correlación de $49 \%$ con GC, expresando su influencia sobre la centralidad ${ }^{7}$. Cuando medimos el indicador de accesibilidad como su potencial (incluyendo barrios cercanos con P2) sin incluir el j evaluado, los resultados muestran que la accesibilidad de los barrios vecinos también modifican a GC en j (Modelo 6, Cuadro 3); sin embargo, al incluir este potencial en el modelo conjunto de oferta y demanda, su significancia se debilita debido a su colinealidad con otras variables (Modelo 5). De esta manera se corrobora que las variables estructurales del modelo conjunto tienen más poder explicativo que la accesibilidad.

7 No se introduce en el modelo de regresión porque el logaritmo natural de esta variable es inexistente para $44 \%$ de los casos que tienen valor cero, reduciendo demasiado el universo de análisis. 
Cuadro 2. Modelos de Regresión. Variable dependiente: Ln Grado de Centralidad GC-E1

\begin{tabular}{|c|c|c|c|c|c|c|c|c|}
\hline \multirow[b]{2}{*}{ VARIABLES } & \multicolumn{2}{|c|}{$\begin{array}{l}\text { Modelo 1: } \\
\text { Oferta }\end{array}$} & \multicolumn{2}{|c|}{$\begin{array}{l}\text { Modelo 2: } \\
\text { Oferta }\end{array}$} & \multicolumn{2}{|c|}{$\begin{array}{l}\text { Modelo 3: } \\
\text { Demanda }\end{array}$} & \multicolumn{2}{|c|}{$\begin{array}{c}\text { Modelo 4: Oferta } \\
\text { y Demanda }\end{array}$} \\
\hline & Coef. & Sig. & Coef. & Sig & Coef. & Sig. & Coef. & Sig. \\
\hline (Constante) & -3.993 & .000 & -2.307 & .000 & 3.045 & .000 & -.937 & .085 \\
\hline Ln Productividad Comercio & .689 & .000 & .242 & .001 & & & .160 & .015 \\
\hline Ln Productividad Servicios & .325 & .001 & .083 & .334 & & & & \\
\hline Ln Densidad de Establecimientos & & & 1.062 & .000 & & & .991 & .000 \\
\hline $\begin{array}{l}\text { Ln_(P2) Proporción de } \\
\text { PEA con } 2 \text { a } 5 \text { SM }\end{array}$ & & & & & -1.591 & .000 & -.616 & .000 \\
\hline $\begin{array}{l}\text { Ln_(P1) Proporción de } \\
\text { PEA con más de } 5 \text { SM }\end{array}$ & & & & & 3.161 & .000 & .880 & .003 \\
\hline Ln Segregación & & & & & -.398 & .004 & & \\
\hline $\mathrm{R} 2$ & .27 & & & & .226 & & & \\
\hline
\end{tabular}

Fuente: elaboración personal con base en datos del Censo de población 2000 y Censos económicos 2004

Cuadro 3. Modelos de Regresión. Variable dependiente: Ln Grado de Centralidad GC-E1

\begin{tabular}{|c|c|c|c|c|c|c|c|c|}
\hline \multirow[b]{2}{*}{ VARIABLES } & \multicolumn{2}{|c|}{ Modelo 5} & \multicolumn{2}{|c|}{ Modelo 6} & \multicolumn{2}{|c|}{ Modelo 7} & \multicolumn{2}{|c|}{ Modelo 8} \\
\hline & Coef. & Sig. & Coef. & Sig. & Coef. & Sig. & & \\
\hline (Constante) & -1.083 & .057 & 2.655 & .000 & -.795 & .151 & 1.550 & .000 \\
\hline Ln Productividad Comercio & .153 & .021 & & & .156 & .018 & & \\
\hline $\begin{array}{l}\text { Ln Densidad de Es- } \\
\text { tablecimientos }\end{array}$ & .986 & .000 & & & .977 & .000 & & \\
\hline $\begin{array}{l}\text { Ln_(P2) Proporción de } \\
\text { PEA con } 2 \text { a } 5 \text { SM }\end{array}$ & -.547 & .005 & & & -.531 & .005 & & \\
\hline $\begin{array}{l}\text { Ln_(P1) Proporción de } \\
\text { PEA con más de } 5 \text { SM }\end{array}$ & .564 & .223 & & & .697 & .031 & & \\
\hline $\begin{array}{l}\text { Ln (P2) Longitud de vías } \\
\text { principales externas }\end{array}$ & .101 & .375 & .822 & .000 & & & & \\
\hline Ln Distancia al centro & & & & & -.112 & .172 & & \\
\hline $\begin{array}{l}\text { Ln Proporción de área con } \\
\text { asentamientos informales }\end{array}$ & & & & & & & -.090 & .024 \\
\hline $\mathrm{R} 2$ & .569 & & .19 & & .57 & & .0 & \\
\hline
\end{tabular}

Fuente: elaboración personal con base en datos del Censo de población 2000 y Censos económicos 2004 
También, desde el modelo monocéntrico y sus derivados policéntrico, a la distancia desde el centro de la ciudad, o desde un subcentro, al lugar de residencia se le ha considerado la mejor aproximación de la intensidad de la actividad económica: al decrecer la distancia se incrementa la densidad de empleo. Esta es una regularidad empírica, grosso modo, que aún se presenta en muchas ciudades (Hajrasoulihaa y Shima Hamidib, 2017), pero que no ha tenido un sólido sustento teórico en gran parte debido a que tanto el modelo monocéntrico como la mayoría de los policéntricos consideran a los centros determinados de manera exógena o solo como lugares de empleo. Dado que está correlacionada con GC, al modelo conjunto se le añade el indicador distancia desde el 'centro' de la ciudad (AGEB con mayor densidad de empleo terciario) hacia cada barrio; el resultado muestra que la significancia estadística de distancia se debilita debido a su colinealidad con otras variables (Modelo 7); de esta manera se corrobora que las variables estructurales del modelo conjunto tienen más poder explicativo que la distancia desde el 'centro'.

Como última prueba, se contrasta GC con asentamientos informales (proporción de su área en el barrio), y se encuentra que la R2 es muy baja para ser considerada una variable determinante de la centralidad (Modelo 8): surgirán centros donde haya demanda aún si las arquitecturas están sobre suelo irregular.

En síntesis, con la excepción de densidad de población y asentamientos informales, la evidencia estadística comprueba que la distribución de las intensidades de centro se puede explicar por factores de oferta y de demanda cercanamente localizada, siguiendo a la TLC intraurbana.

Estos resultados del estudio permiten deducir dos consecuencias urbanísticas. La primera se refiere al efecto de la estructuración de centros sobre la redistribución del ingreso per- sonal. Centros de mayor jerarquía se localizan cerca de barrios con residentes de alto ingreso; cerca de los barrios con población de bajo ingreso solo aparecen centros de baja jerarquía o no hay centros. Las personas con bajo ingreso recibido en su trabajo deben además gastar más en transporte para acudir a consumir o trabajar, comparados con las personas de más ingreso. El patrón espacial -y la estructuraciónde la jerarquía de centros generan una redistribución regresiva del ingreso.

En contraste, la segunda consecuencia es positiva para la planeación. Por el modo en que se derivó el Índice de centralidad de una actividad en un barrio, $C_{i j}$, este índice expresa la intensidad de la actividad en un lugar, intensidad medida con una tasa relativa: $\left(E_{i j} / E_{i}\right) /\left(P_{j} / P\right)$. Si la actividad $i$ llegara a ser central en un barrio j $\left(C_{i j}>1\right)$, j tendría un $\mathrm{GC}_{\mathrm{j}} \geq 1$ y con ello, una sobre proporción de empleo i (respecto a su población), propiciando así una mayor densidad de empleo de esa actividad en el barrio $\left(E_{i j} / \mathrm{Ha}\right)$. Esto nos permite deducir que a medida que haya más actividades centrales en un barrio, es decir mayor GC, ahí se incrementará la densidad total de empleo. Con esta deducción se implementó una regresión donde GC determina la densidad de empleo terciario, cuyo resultado se puede ver en el Gráfico 1.

La bondad de ajuste de la regresión es $72 \%$ y podría ser mayor (81\%) si omitimos el caso extremo en la parte alta a la derecha en el Gráfico 1. Este resultado se corrobora si comparamos la distribución del Grado de centralidad (Mapa 1) con la distribución espacial de la densidad de empleo terciario (Mapa 2).

Este resultado es relevante desde el punto de vista de la planeación urbana. La especificación de centros según su grado de centralidad solo requiere información por barrio (o AGEB) de la densidad de empleo, de comercio y servicios, y tal especificación estaría sustentada por la teoría del lugar central intraurbana que aquí se expuso y comprobó. 


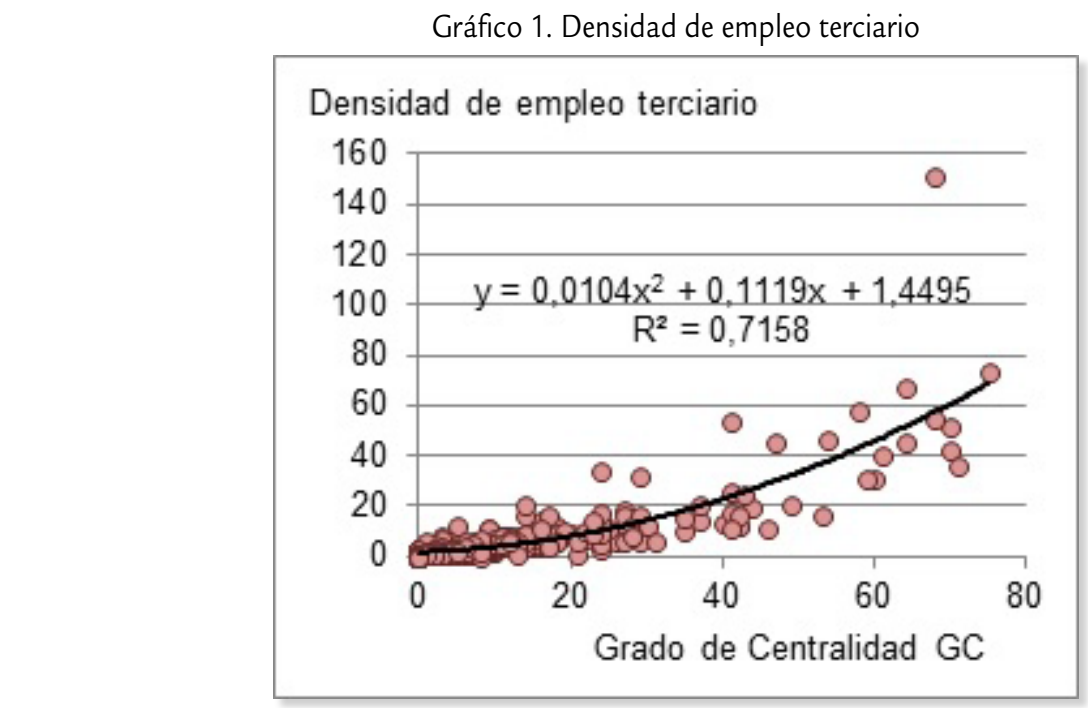

Fuente: elaboración personal con base en datos del Censo de población 2000 y Censos económicos 2004

Mapa 2. Tijuana: Densidad de empleo terciario

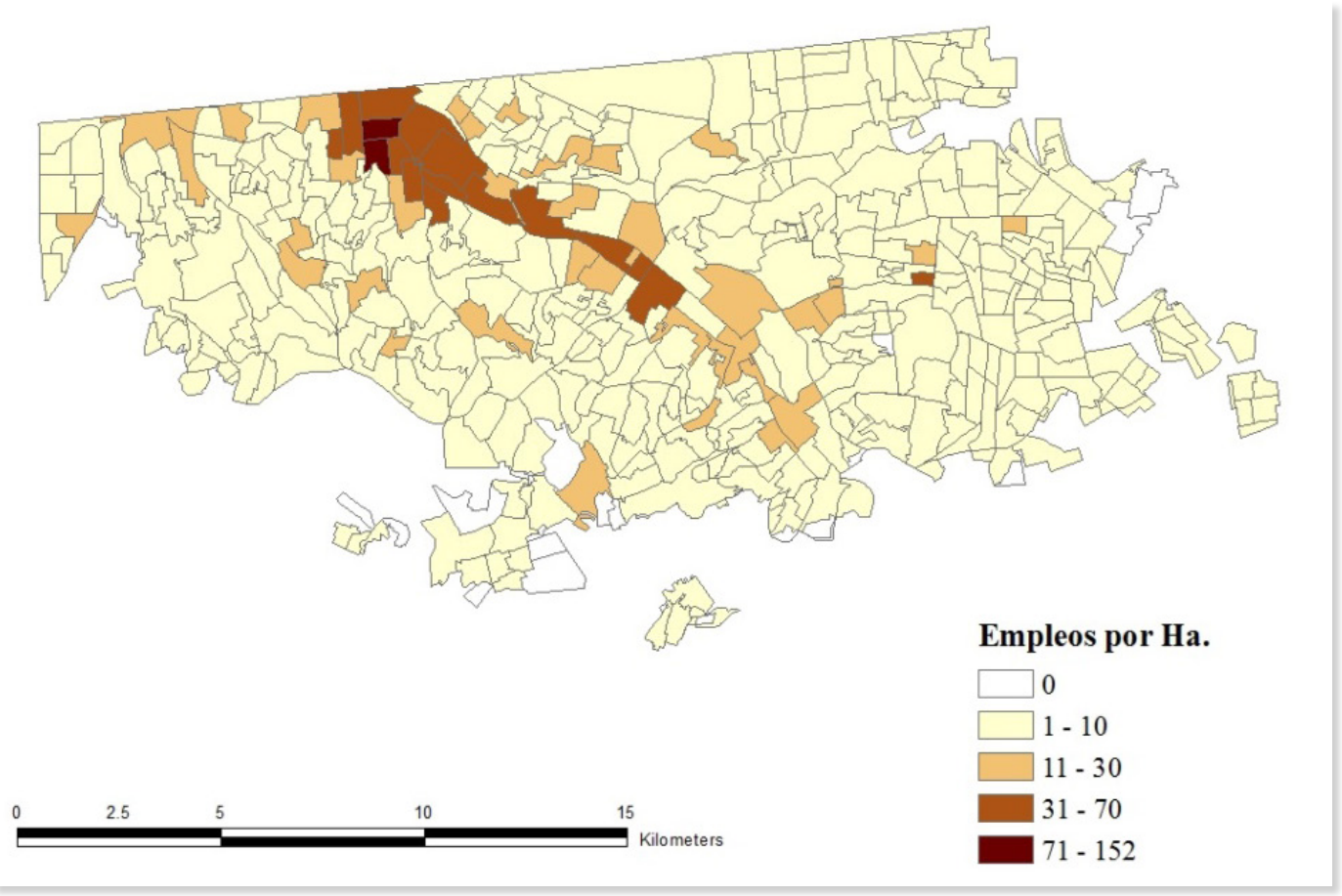

Fuente: elaboración personal con base en datos de Censos económicos 2004

\section{COMENTARIOS FINALES}

Considerar centro como concentración de empleos ha limitado el desarrollo de la teoría por varias razones, entre ellas tres principales. Primero, se incluyen actividades terciarias mez- cladas con las de industria como si tuvieran el mismo patrón espacial y los mismos factores de localización. El objetivo de localización del terciario es incrementar las ventas (depende de la demanda final localizada), en contraste, el 
de la industria es reducir los costos (pagando menos por suelo periférico y por otros insumos localizados). Segundo, bajo el enfoque prevaleciente de economía espacial el lado de la demanda ha estado ausente en la definición y explicación de los centros a pesar que el terciario depende del consumo final (mientras que la industria no). Y tercero, de manera irreal, se ha considerado usos del suelo segregados en el espacio al incluir solo actividades económicas (empleo) en los barrios centrales.

Definiendo centro como lugares de actividad, aquí se ha formulado una teoría de la distribución espacial de intensidades de la actividad terciaria, que permite definir dónde y con qué grado aparece un centro. Esta teoría considera que los centros son determinados por factores de oferta y demanda operando conjuntamente en el espacio, en un medio de usos mixtos del suelo. Para llevar a cabo la comprobación empírica de esta teoría se derivó una definición analítica de centro, y se implementó una prueba estadística. Los resultados indican que la teoría tiene una gran corroboración empírica con los datos de la ciudad de Tijuana.

Falta, sin embargo, desarrollar dos aspectos del modelo. Primero, la derivación de Cij se hizo con factores de demanda, sin embargo no se incluyó indicadores de intercambio intermedio (relaciones hacia adelante y atrás tipo insumo-producto) ni de difusión de conocimiento, ambos importantes en la reducción de costos debido a la co-localización. Segundo, los datos usados en este estudio son de actividades formales por lo que se supone que la informal sigue un patrón espacial similar a las primeras; este supuesto se podría confirmar si hubiera datos adecuados.

\section{REFERENCIAS}

Aguilar, A.G. y Alvarado, C. (2005). La reestructuración del espacio urbano de la Ciudad de México ¿Hacia la metrópoli multinodal? (pp. 265307). En A.G. Aguilar (Ed.) Procesos metropolitanos grandes ciudades. Dinámicas recientes en México y otros países. México: Miguel Angel Porrúa.

Alegría, T. (1994). Segregación socioespacial urbana. El ejemplo de Tijuana. Estudios demográficos y urbanos, 9(2), 411-428.

Alegría, T. (1997). Centrality and location of employment in greater Lima (pp. 199-225). En PromPerú (Ed.) Peru: Beyond the reforms. PromPerú summer internship program 1996. Lima: Imagen y Medios.

Alegría, T. (2009). Metrópolis transfronteriza. Revisión de la hipótesis y evidencias de Tijuana, México y San Diego, Estados Unidos. México: Editorial Miguel Ángel Porrúa y El Colef.

Alonso, W. (1964). Location and land use. Cambridge, MA: Harvard University Press.

Anas, A. y Kim, I. (1996). General equilibrium models of polycentric urban land use with en-

dogenous congestion and job agglomeration. Journal of Urban Economics, 40(2), 232-256.

Anas, A.; Arnott, R. y Small, K. (1998). Urban spatial structure. Journal of Economics Literature, 36(3), 1426-1464.

Anderson, N. y Bogart, W. (2001). The structure of sprawl: Identifying and characterizing employment centers in polycentric metropolitan areas. American Journal of Economics and Sociology, 60(1), 147-169.

Arribas-Bel, D. y Sanz-Gracia, F. (2014). The validity of the monocentric city model in a polycentric age: US metropolitan areas in 1990, 2000 and 2010. Urban Geography, 35(7), 980-997.

Batty, M. (2017). Science in Planning: Theory, Methods and Models (pp. 241-254). En T. Sanchez (Ed) Planning Knowledge and Research. New York, USA: Routledge.

Baumol, W.J. (1959). The Revenue Maximization Hypothesis (pp. 45-53). En Business Behavior, Value, and Growth. New York: Macmillan.

Berry, B. y Parr, J. (1988). Market centers and retail 
location. Englewood Cliff, NJ: Prentice Hall.

Berry, B. (1963). Commercial structure and commercial blight: retail patterns and processes in the city of Chicago. Chicago: Dept. of Geography, University of Chicago. Assisted by R.J. Tennant, B.T. Garner, J. W. Simmons. Recuperado de https://babel.hathitrust.org/ cgi/pt?id=uc1.b3377010\&view=1up\&seq $=5$ (consulta diciembre 2019).

Bogart, W. y Ferry, W. (1999). Employment centres in greater Cleveland: Evidence of evolution in a formerly monocentric city. Urban Studies, 36(12), 2099-2110.

Bourdieu, P. (1990). Sociología y Cultura. México: Grijalbo y CONACULTA.

Brinkman, J.C. (2016). Congestion, agglomeration, and the structure of cities. Journal of Urban Economics, 94(July), 13-31.

Burger, M. y Evert, M. (2012). Form Follows Function? Linking Morphological and Functional Polycentricity. Urban Studies, 49(5), 1127-1149.

Cervero, R. y Wu, K-L. (1997). Polycentrism, commuting, and residential location in the San Francisco Bay área. Environment and Planning A, 29(5), 865-886.

Christaller, W. (1966). Central Places in Southern Germany. Englewood Cliffs: Prentice Hall (traducido por C. W. Baskin de la versión original de 1933 en alemán).

Eaton, C. y Lipsey, R. (1982). An economic theory of central places. The Economic Journal, 92(365), 56-72.

Fernández-Maldonado, A.M.; Romein, A; Verkoren, O. y Parente Paula Pessoa, R. (2014). Polycentric structures in Latin American metropolitan areas: Identifying employment sub-centres. Regional Studies, 48(12), 1954-1971.

Forstall, R. y Greene, R.P. (1997). Defining job concentrations: The Los Angeles case. Urban Geography, 18(8), 705-739.

Förster, M. y Pearson, M. (2002). Income distribution and poverty in the OECD area: Trend and driving forces. OECD Economic Studies, (1), 7-39.
Fujita, M. y Ogawa, H. (1982). Multiple equilibria and structural transition of non-monocentric urban configurations. Regional Science and Urban Economics, 12(2), 161-196.

Galeano, V. (2013). Localización espacial de la actividad económica en Medellín, 2005-2010. Un enfoque de economía urbana. Ensayos sobre Política Económica, 31(70). (Edición especial sobre economía de las ciudades)

Giuliano, G. y Small, K. (1991). Subcenters in the Los Angeles region. Regional Science and Urban Economics, 21(2), 163-182.

Gonzales de Olarte, E. y del Pozo, J.M. (2012). Lima, una ciudad policéntrica. Un análisis a partir de la localización del empleo. Investigaciones Regionales, (23), 29-52.

Gordon, P. y Richardson, H.W. (1996). Beyond polycentricity: The dispersed metropolis, Los Angeles, 1970-1990. Journal of the American Planning Association, 62(3), 289-295.

Graizbord, B. y Acuña, B. (2005). La Estructura Polinuclear del Area Metropolitana (pp. 309328). En A. Aguilar (Ed.) Procesos Metropolitanos y Grandes Ciudades. Dinámicas Recientes en México y otros Países. México: Porrúa.

Graizbord, B. y Santillán, M. (2005). Dinámica demográfica y generación de viajes al trabajo en el AMCM: 1994-2000. Estudios Demográficos y Urbanos, 20(1), 71-101.

Hajrasoulihaa, A.H. y Hamidib, S. (2017). The typology of the American metropolis: monocentricity, polycentricity, or generalized dispersion? Urban Geography, 38(3), 420-444.

Henderson, V. y Slade, E. (1993). Development games in non-monocentric cities. Journal of Urban Economics, 34(2), 207-229.

Manaugh, K. y Kreider, T. (2013). What is mixed use? Presenting an interaction method for measuring land use mix. Journal of Transport and Land Use, 6(1), 63-72.

McDonald, J.F. (1987). The identification of urban employment subcenters. Journal of Urban Economics, 21(2), 242-258.

McMillen, D. (2001). Nonparametric employment subcenter identification. Journal of Urban 
Economics, 50(3), 448-473.

McMillen, D. y McDonald, J.F. (1998). Suburban subcenters and employment density in metropolitan Chicago. Journal of Urban Economics, 43(2), 157-180.

Mert, M. (2018). What does a firm maximize? A simple explanation with regard to economic growth. International Journal of Engineering Business Management, 10, 1-13.

Montejano, J.; Caudillo, C. y Silván, J. (2016). Contesting Mexico City's alleged polycentric condition through a centrality-mixed land-use composite index. Urban Studies, 53(11), 2380-2396.

Muñiz, I.; Sánchez, V. y García-López, M-A. (2015). Estructura espacial y densidad de población en la ZMVM 1995-2010: evolución de un sistema urbano policéntrico. EURE, 41(122), 75-102.

Parkin, M.; Esquivel, G. y Ávalos, M. (2006). Microeconomía: Versión para Latinoamérica. México: Pearson Educación.

Redfearn, C.L. (2007). The topography of metropolitan employment: Identifying centers of employment in a polycentric urban area. Journal of Urban Economics, 61(3), 519-540.

Richardson, H.W. (1977). The new urban economics and alternatives. London: Pion Limited.

Richardson, H.W. (1988). Monocentric vs. policentric models: The future of urban economics in regional science. The Annals of Regional Science, 22(2), 1-12.

Rodriguez-Gámez, L. y Dallerba, S (2012). Spatial distribution of employment in Hermosillo 1999-2004. Urban Studies, 49(16), 3663-3678.

Rojas, D. (2015). Localización de centros de empleo y su influencia sobre la distribución de la población en el Distrito Metropolitano de Quito. Analítika, 9(1), 53-93.

Ross, S. y Yinger, J. (1995). Comparative static analysis of open urban models with a full labor market and suburban employment. Regional Science and Urban Economics, 25(5), 575-605.

Schulz, N. y Stahl, K. (1996). Do consumers search for the highest price? Oligopoly equilibrium and monopoly optimum in differentiated-products markets. The RAND Journal of Economics, 27(3), 542-562.

Seale, J.L.; Regmi, A. y Bernstein, J.A. (2003). International evidence on food consumption patterns (Technical bulletin no. 1904). Washington, DC: Economic Research Service/USDA. Recuperado de https://ageconsearch.umn.edu/ bitstream/33580/1/tb031904.pdf (consulta 10 de mayo 2019)

Stahl, K. (1987). Theories of urban business location (pp. 759-820). En E. Mills (ed.) Handbook of regional and urban economics, 2. Amsterdam: North-Holland.

Suárez, M. y Delgado, J. (2009). Is Mexico City polycentric? A trip attraction capacity approach. Urban Studies, 46(10), 2187-2211.

Suárez, M.; Murata, M. y Delgado, J. (2016). Why do the poor travel less? Urban structure, commuting and economic informality in Mexico City. Urban Studies, 53(12), 2548-2566.

Sullivan, A.M. (1986). A general equilibrium model with agglomerative economies and decentralized employment. Journal of Urban Economics, 20(1), 55-74.

Usach, N.; Garrido-Yserte, R. y Gallo-Rivera, M-T. (2017). Organización territorial y funcional de la metrópoli de Buenos Aires. EURE, 43(128), 55-80.

van Meeteren, M.; Poorthuis, A.; Derudder; B y Witlox, F. (2016). Pacifying Babel's Tower: A scientometric analysis of polycentricity in urban research. Urban Studies, 53(6), 1278-1298.

West, D.; Von Hohenbalken, B. y Kroner, K. (1985). Tests of intraurban central place theories. The Economic Journal, 95(377), 101-117.

Wheaton, W.C. (2004). Commuting, congestion, and employment dispersal in cities with mixed land use. Journal of Urban Economics, 55(3), 417-438.

White, M. (1999). Urban areas with decentralized employment: Theory and empirical work 
(pp. 1375-1412). En E. Mills y P. Cheshire (Eds.) Handbook of regional and urban economics, 3. New York, NY: North-Holland.

Wieand, K. (1987). An extension of the mo- nocentric urban spatial equilibrium model to a multicenter setting: The case of the two-center city. Journal of Urban Economics, 21(3), 259-271.

Tito Alejandro Alegría Olazábal es Doctor en Planeación y Desarrollo Urbano (University of Southern California, Los Angeles, EEUU). Maestro en Desarrollo Urbano (El Colegio de México). Arquitecto (Universidad Nacional de Ingeniería, Lima, Perú). Investigador de El Colef, desde 1987, e integrante del Sistema Nacional de Investigadores, SNI, nivel 2. Profesor de las sub-disciplinas urbanas de economía, sociología y geografía. Ha publicado 5 libros y más de 50 artículos académicos en 10 países sobre: crecimiento urbano, trabajo transfronterizo, sub-centros de comercio y servicios, segregación, regularización del suelo, precio y uso del suelo, y sobre integración urbana de extranjeros. Su último libro de autor es "Metrópolis Transfronteriza: Revisión de la hipótesis y evidencias de Tijuana, México y San Diego, Estados Unidos". Ha tenido estancias de investigación o docencia en Perú, Estados Unidos, Italia, México y Francia, y ha sido consultor de gobiernos locales y del programa HABITAT de la ONU. Departamento de Estudios Urbanos y del Medio ambiente. El Colegio de la Frontera Norte (EI Colef). Carretera escénica Tijuana. Ensenada, Km 18.5, San Antonio del Mar (C.P. 22560) Tijuana, Baja California, México, talegria@colef.mx 